\title{
Deploying the ICT Architecture of a Residential Demand Response Pilot
}

\author{
Matthias Strobbe $^{1}$, Koen Vanthournout ${ }^{2}$, Tom Verschueren ${ }^{1}$, Wim Cardinaels $^{2}$ and Chris Develder ${ }^{1}$ \\ ${ }^{1}$ Ghent University - iMinds \\ Dept. of Information Technology - IBCN, Ghent, Belgium \\ Email: matthias.strobbe@intec.ugent.be \\ ${ }^{2}$ VITO-EnergyVille \\ Mol, Belgium \\ Email: koen.vanthournout@vito.be
}

\begin{abstract}
The Flemish project Linear was a large scale residential demand response pilot that aims to validate innovative smart grid technology building on the rollout of information and communication technologies in the power grid. For this pilot a scalable, reliable and interoperable ICT infrastructure was set up, interconnecting 240 residential power grid customers with the backend systems of energy service providers (ESPs), flexibility aggregators, distribution system operators (DSOs) and balancing responsible parties (BRPs). On top of this architecture several business cases were rolled out, which require the sharing of metering data and flexibility information, and demand response algorithms for the balancing of renewable energy and the mitigation of voltage and power issues in distribution grids. The goal of the pilot is the assessment of the technical and economical feasibility of residential demand response in real life, and of the interaction with the end-consumer.

In this paper we focus on the practical experiences and lessons learnt during the deployment of the ICT technology for the pilot. This includes the real-time gathering of measurement data and real-time control of a wide range of smart appliances in the homes of the participants. We identified a number of critical issues that need to be addressed for a future full-scale roll-out: (i) reliable in-house communication, (ii) interoperability of appliances, measurement equipment, backend systems, and business cases, and (iii) sufficient backend processing power for real-time analysis and control.
\end{abstract}

Keywords-Smart Grids, Residential Demand Response, ICT architecture, Pilot, Practical Experiences

\section{INTRODUCTION}

In recent years there has been a shift from centralized, power generation, based on fossil and nuclear fuels, to distributed, renewable power generation. Environmental concerns and efforts to reduce dependence on fossil fuels are the driving force for the replacement of traditional energy sources by green alternatives. The intermittent nature of renewable energy sources, such as solar or wind, makes it difficult to balance demand and supply, which is essential for the correct operation of the power grid. Additionally, electricity demand is rising, e.g., as a result of the ongoing electrification of the vehicle fleet and the adoption of heat pumps. Also, investments in controllable (fossil-fuel, typically gas-fired) plants are decreasing due to a low profitability, as power from renewable energy sources gets priority on the grid.

Intermittent production can be compensated for by deploying storage and by adapting the demand to the availability of (renewable) production: demand response. Whereas demand response (DR) is increasingly deployed in the industry, the large potential in the residential and tertiary sector remains until now unused. Residential settings have specific properties and constraints: preserving the user's comfort is of prime importance to achieve sustained participation in demand response; individual flexibility sources only contribute a small amount of energy, but they are large in number, requiring scalable and low cost control and communication technologies. As such, the technology needed for residential DR is different from the industrial equivalent and requires the extended implementation of scalable information and communication technology (ICT) into the power grid domain.

The goal of the Flemish smart grid project Linear ${ }^{1}$ [1] was to investigate the technical and economical feasibility of residential demand response technology as well as analyze user behaviour and acceptance. Therefore a large-scale pilot with 240 households was set up to test the technology in the field and to evaluate different business cases. The Linear pilot distinguishes itself from other pilots on residential demand response, e.g., [2]-[12] by the integration of a wide array of different types of appliances. Additionally, other pilots typically focus on a single or limited set of control/business cases, and are often vertically integrated, i.e., each link in the architecture is realized by a single company and/or component. Linear takes a wider scope by supporting appliances from multiple vendors and by supporting multiple energy service providers. This wider scope also translates to the size of the pilot; in terms of number of participants with smart appliances and number of smart appliances per residence, Linear ranks among the most ambitious, which makes the Linear residential DR pilot one of the most complete today.

In [13] we presented the ICT infrastructure that was designed for the pilot. In this paper we focus on the practical

\footnotetext{
${ }^{1}$ http://www.linear-smartgrid.be/?q=en
} 
experiences and lessons learnt when deploying and managing the different blocks of the architecture in the field. In Section II we provide more details on the setup and goals of the pilot, followed by an overview of the designed architecture in Section III. Section IV is the core of the paper and discusses our practical experiences with the deployment in the field. Finally, conclusions are stated in Section V.

\section{PILOT}

Within Linear four business cases are evaluated technically and economically:

- Portfolio Management: Can we use day ahead dynamic tariffs to make customers shift their energy consumption towards off-peak hours and periods with an abundant amount of renewable energy?

- Wind Balancing: Can we reduce intra-day imbalance costs for a BRP, caused by the deviation between predicted and produced wind energy?

- Transformer Aging: Can we reduce the aging of transformers by shifting the local energy consumption to avoid high transformer temperatures?

- Line Voltage Management: Can we avoid too large voltage deviations in local grids, for example caused by the injection of a high amount of solar energy on sunny summer days or by the simultaneous charging of lots of electric vehicles in the evening?

The pilot consists of 240 households, partitioned in two subsets. 130 households are geographically spread over the region of Flanders. These users participate in the experiments with a commercial focus (portfolio management, wind balancing). The second subset of 110 users are living close together in two neighbourhoods so that several users are connected to the same feeder and transformer. This allows to test the location specific, technical cases (transformer aging, line voltage management). In those neighbourhoods also wind balancing is tested, to assess its effect on the local distribution grid.

In a first stage, measurement devices (residence metering or smart meters, and smart plugs for submetering) were installed in the houses to get measurements of household consumption and production (94 households have photo-voltaic panels, representing a total of $400 \mathrm{kWp}$ ) and detailed appliance consumption. In total, about 2000 submetering points are installed and 110 households are equipped with smart meters. In the first phase of the project, these measurements were purely used for monitoring, and to gather baseline reference data. In the second phase, during the tests of the business cases, the measurements were also used as inputs for control (DR), and in the evaluation thereof (e.g., comparing them to the reference data).

During the second phase, post the reference measurements, remotely controllable smart appliances were installed in 185 households. Two types of smart appliances, that offer large amounts of flexibility and that can be automated were selected to minimize the comfort impact for the participants. The first type consists of appliances, such as dishwashers, washing machines and tumble dryers. A total of 460 deferrable appliances were deployed in the pilot. The second type comprises appliances with buffers, such as domestic hot water buffers (DHW, 15 deployed) and electrical vehicles (EV, 7 deployed).

Every participant received a home gateway which interacts with the measurement equipment and smart appliances, and a display in the form of a tablet to get insight in their consumption patterns, as well as the amount of provided flexibility and associated financial bonus. Users are asked to provide as much flexibility as possible when configuring their smart appliances, e.g., by configuring the devices before going to work with a deadline at the time they return home.

Two types of interaction models are used in the pilot:

- Day ahead dynamic prices [14] are tested with the 55 families without smart appliances. A day is divided in 6 fixed blocks (night, morning, noon, afternoon, evening, late evening), but the tariffs differ from day to day and are based on the day ahead power market, scaled-up to 2020 wind and solar production predictions. The users can consult these tariffs on their display from 4 PM the day before. If they shift consumption from expensive hours to cheap moments (in comparison with their reference measurements), they get a financial reward. This model supports the business case of day ahead portfolio management.

- The second model is based on the flexibility offered by the participants with smart appliances. This flexibility is used in experiments for all four business cases. The participants get a financial reward based on the amount of provided flexibility.

\section{Pilot ARChitecture}

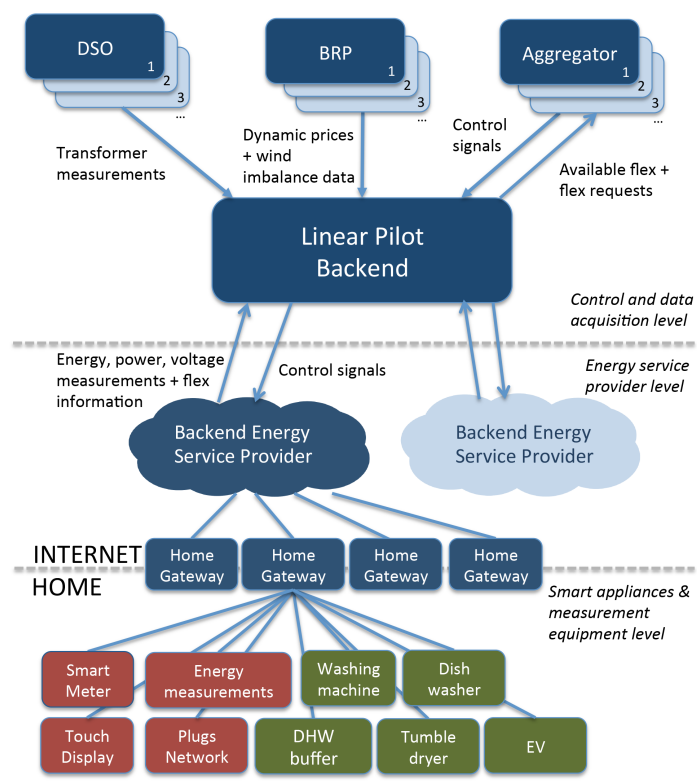

Fig. 1. Overview of the ICT architecture for the Linear pilot.

Figure 1 shows the ICT architecture that was designed, implemented and deployed for the pilot. An important requirement was the need to support multiple actors (energy service providers, flexibility aggregators, BRPs, DSOs), each with their own proprietary system. Also support was needed 
for smart appliances and smart meters from multiple vendors using different communication technologies. Furthermore the real-time collection of consumption, production and flexibility data both on household and on appliance level was essential for the evaluation of the commercial and technical business cases outlined in section II.

The resulting architecture consists of three layers with clear interfaces in between the layers and special focus on the device abstraction of the smart appliances. The architecture proved a success, as all components and functionalities were successfully integrated, despite several scope changes in the course of the project. For instance, the electrical vehicles were only added while the pilot was already operational. All measured data is collected centrally in a data management system to allow a real-time follow-up of the user setups and in depth analyses of the operation of the system, the interaction with the participants and the business case experiments.

All appliances installed at a residence are connected to and aggregated via a home gateway managed by a Energy Service Provider (ESP). Measured consumption, production and flexibility data is sent in real-time to the backend of the ESP and from there to the pilot backend. Inputs and requests for using the available flexibility are sent from the BRP and DSO partners in the form of wind imbalance setpoints, dynamic price information and real-time measurements from 5 transformers in the field (current, voltage, phase and temperature) to the central backend and from there to the server of the flex aggregator. This flex aggregator aggregates flexibility data from the smart appliances and uses this flexibility to support the outlined business cases. The flex requests are matched with the currently available flex, resulting in the generation of a number of control signals (e.g., to start a number of white goods or stop charging an EV). These signals are sent via the pilot and ESP backends to the home gateways and from there to the smart appliances. Users without smart appliances receive the dynamic prices from the pilot backend through the ESP backend, onto their tablets. Similarly, users can request the status of their financial bonuses.

\section{DEPloyment \& PRACTICAL EXPERIEnCES}

In this section we focus on the practical experiences and lessons learnt when implementing and deploying the pilot infrastructure. Most attention goes to the bottom (in-house) and top layer (pilot backend) of the architecture. The middle layer consists of proprietary systems of the energy service provider companies.

As a wide and diverse range of systems and appliances are combined, testing of the whole chain before going to the field is key. Therefore different labs were set up, ranging from test facilities for testing the control of individual appliances, over the installation of complete setups in imitations of real residences, and the deployment of a few setups in homes of people directly involved with the implementation of the pilot. These lab setups allowed to perform validation and integration tests, testing the setups in real environments, evaluating the correct operation of the different business cases and reconstructing problems reported by field trial participants. Tests involving different labs allowed to compare results, test scenarios over a small cluster of imitated households and an assessment of the test scenarios from the specific perspective and experience of the different partners.

\section{A. Smart appliances and in-house communication}

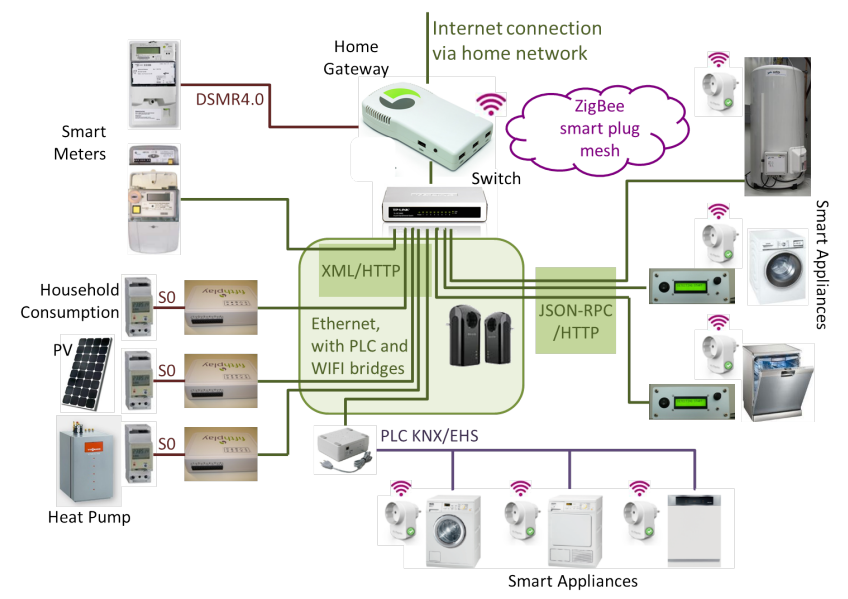

Fig. 2. Overview of the Linear in-house communications.

The heart of the Linear setup in all households is the Home Energy Management Gateway (see Figure 2). The gateway is connected to all the other in-house equipment and collects all measurements and status data, pushes this data via its broadband internet connection to the Linear backend, and dispatches demand respond control actions from the backend to the smart appliances. Various technologies are used to set up communications between gateway and the other in-house components (see Figure 2):

The smart appliances:

- The smart DHW buffers, and one brand of dishwashers and washing machines, both rendered smart via an external controller, support a JSON-RPC/HTTP interface over Ethernet.

- $\quad$ The second brand of dishwashers, washing machines and tumble dryers, all with on-board demand response functionality, are controlled via a separate gateway that connects to the smart appliances via power line communications, more specifically KNX/EHS [15]. The appliance gateway in turn connects to the home energy management gateway via an XML/HTTP interface over Ethernet.

Measurements:

- Two types of smart meters are deployed in the Linear pilot. The first type connects to the gateway via the wired DSMR 4.0 protocol [16], which was extended to include voltage measurements. The second type supports an XML/HTTP interface over Ethernet.

- In the absence of smart meters, a separate device is installed to measure the overall electricity consumption of the household. A small hub converts the energy pulses of that meter to an XML/HTTP over Ethernet interface. The same measurement setup is deployed for any photovoltaic panels or heat pumps. 
- Each Linear household is equipped with a set of 10 submetering plugs. These are installed at all the smart appliances and the other large consumers in the household. Communication with the gateway is realized via a ZigBee meshed wireless network.

In virtually all installations, it was not feasible to have the Ethernet connections between the smart appliances, smart meters and total consumption/production measurement devices on one hand and the Home Energy Management Gateway on the other hand to be wired (via UTP cables), as Linear installations are all retrofits. In those cases, power line communication (PLC) bridges were deployed. WiFi bridges served as a backup technology in case the PLC did not operate reliably.

The in-house communications proved to be one of the major technical weak points in the Linear infrastructure, as illustrated in Figure 3. Power line communication showed intermittent failures in a large number of houses, with a clear correlation to longer distances and the use of multiple parallel PLC systems. The latter was often unavoidable, as it is inherent to one of the Linear whitegood appliance brands, and as a lot of Flemish households use PLC to connect the television to the internet provider's gateway. WiFi often was no viable alternative: due to the abundant use of stone, concrete and iron in Flemish dwellings, combined with the access points to the appliances typically being next to or behind the metal encasing of those appliances, WiFi was often not able to cover the distance from the appliances to the ISP access point. Note that this ISP access point and the various smart appliances are often located in outlying corners of the house, such as cellars, attics, garages, etc. The same factors reduced the reliability of the ZigBee plugs. ZigBee uses lower transmission power, which should be compensated by its meshed network technology. However, the sparseness of the network (default 10 plugs), combined with the attenuating factors explained above, frequently made it necessary to deploy extra plugs solely for the purpose of creating extra bridges in the network. As this requires power plugs being available at the correct bridging locations, even extra plugs proved insufficient in a few locations. This meant that in practice only skilled and trained technicians were able to set up the customized communication networks. Even then, for a few locations it was not possible to reach full connectivity for all components.

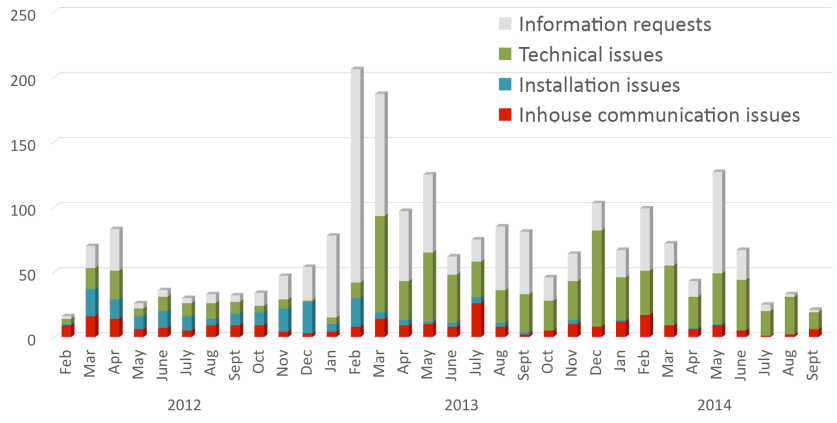

Fig. 3. The number of Linear user support tickets per month, sorted into four categories: requests for information (e.g., on contracts, how-tos, etc.), communication on installations, issues concerning in-house communication failures, and all other technical issues. (e.g., remuneration calculation issues, gateway crashes)
Also noteworthy is that the very large majority of the issues classified as 'other technical issues' in Figure 3, relate to malfunctions of those components supporting the communication functions, such as gateways, PLC electronics embedded in whitegood appliances, etc. The communication and remote control capabilities do encompass the bulk of the new and largely untested functionality deployed in Linear.

Because of this, two of the Linear industrial partners have replaced or drastically adapted their communication technology, e.g., with RF wireless. Conclusion is that one of the major technological gaps that need be addressed before full scale rollout of residential demand response is feasible, is reliable and easy installable in-house communication technology that can be used to retrofit existing dwellings.

\section{B. Set-up and operational management of the pilot backend}

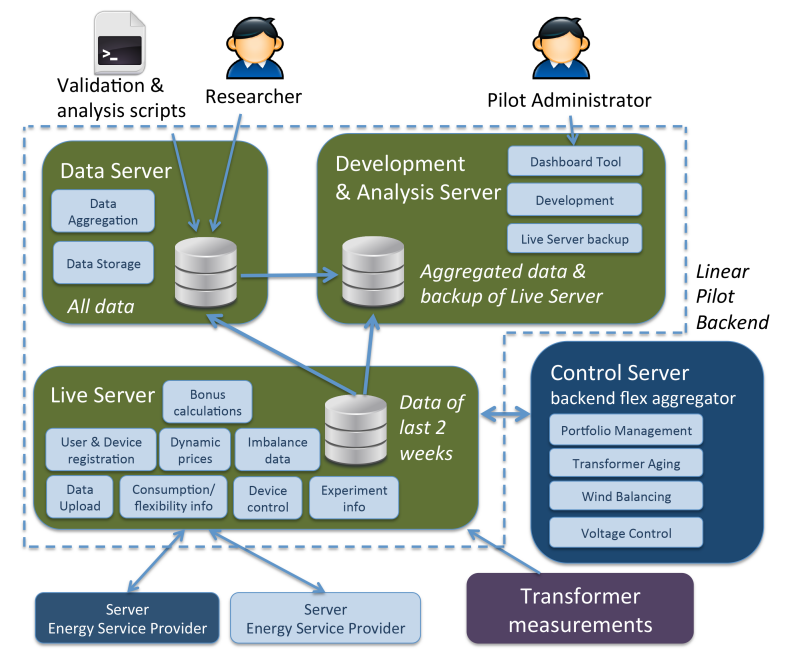

Fig. 4. Overview of the Linear backend.

Figure 4 gives an overview of the setup of the pilot backend, which consists of several servers to meet the imposed requirements. The Live Server is responsible for the real-time gathering of data, the registration of new users, providing relevant information to the end users (e.g., bonuses) and to the business logic of the flex aggregators (e.g., wind imbalance data), and forwarding of control signals to the participants. Standard web service technology and clear interfaces are used for the communication with the backends of the energy service providers and flexibility aggregators. There is also a direct communication link via GPRS to 5 transformers in the field. To guarantee a high availability this server is hosted in a professional datacenter, so that no data is lost nor an experiment is interrupted.

This Live Server continuously receives data from the installations in the field: over 2000 measurement devices send measurement and status data every quarter and over 480 smart appliances send status information when used. This data needs to be stored and made available to interested parties (especially the control algorithms from the flex aggregator) without any delay. Detailed analyses and processing of the data should therefore be executed on another server. In our setup, a second server, the Data Server takes care of these tasks. It contains 
all data received during the complete pilot to allow in-depth analyses of the ongoing and finished experiments.

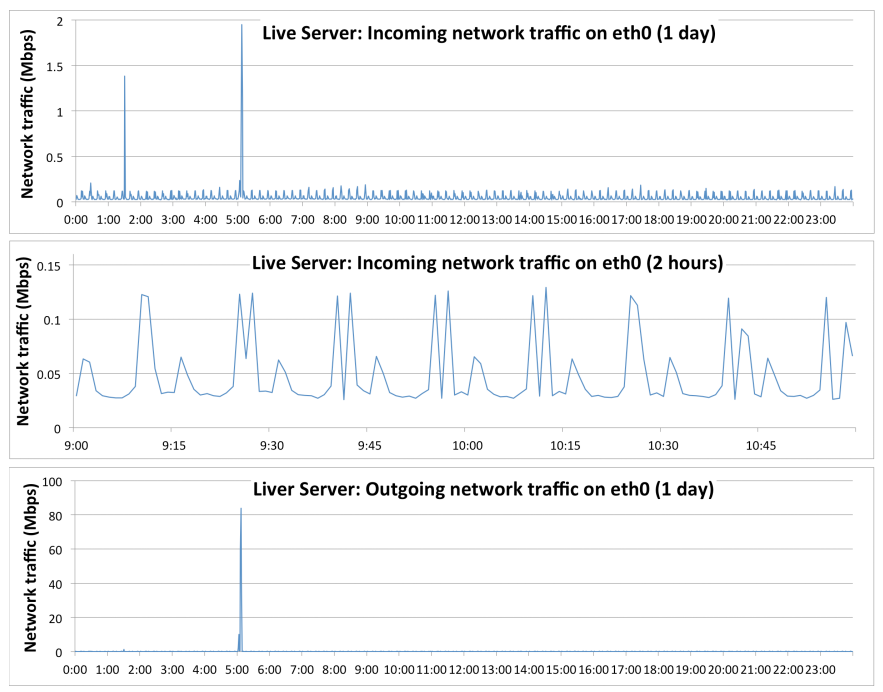

Fig. 5. Overview of incoming and outgoing data on the Live Server.

Figure 5 shows incoming and outgoing network traffic on the Live server. Looking at the incoming traffic (top and middle figure) we see small peaks every quarter as most measurement data is reported on a quarterly basis. There is a short, large peak in the beginning of the night when a daily recalculation of all user bonuses (executed on the Data Server) is uploaded to the Live Server. The large peak on the outgoing network interface and associated peak on the incoming network interface around $5 \mathrm{~h}$ are due to a server backup process. An extra daily backup process of the database caused delays on the handling of incoming data, hindering running experiments and needed to be removed. As solution all data from the Live server was also replicated in real-time on a third server, the Development \& Analysis Server, identical to the Live Server, where a daily backup is made. This server was initially used to implement and test new features and interfaces during the reference measurement period and first experiments.

As database technology we opted for standard MySQL which is free and widely used with lots of tools and documentation available. The master-slave replication mechanism of MySQL [17], [18] is used to send all received data in realtime to the Data Server and Development \& Analysis Server.

Real-life data is never perfect and certainly not in case of an experimental setup where one of the goals is testing the technical feasibility of interconnecting several (prototype) systems to realize residential demand response. Therefore a set of processing scripts are executed on the Data Server to clean, validate and calculate additional data. A few examples:

- Household consumption data is gathered from various measurement setups (see Figure 2). This data is presented in different formats and processing is needed to transform the data into a single net consumption and net PV production value.

- Invalidation of data is needed when data is missing (e.g., due to local communication issues), is com- pletely incorrect (e.g. extreme values from faulty appliances), has missing timestamps, etc.

As Linear deploys an experimental setup, fault detection and handling is important. To mitigate issues fast and minimize the impact on the ongoing experiments, real-time monitoring of the infrastructure is key. The three servers that make up the central backend are monitored via Zabbix [19], [20] and alarms are sent to the pilot administrators when, e.g., a server or connection is down, a replication mechanism is disturbed, memory or hard disk issues occur, etc.

To mitigate in-house communication problems discussed in Section IV-A the home gateway software was gradually extended to provide the backend with more and more status data on the deployed measurement devices and smart appliances. This of course has an impact on the amount of transmitted data and required storage capacity of the Data Server which needed an upgrade during the pilot. Furthermore, we noticed that some limits of standard MySQL technology were reached: replication was not always as reliable as expected. When the Data Server was under high disk load due to ongoing analysis, the replication process had great difficulties in writing the updates to the slave, which on some occasions halted the entire replication process. Due to the high volume of measurements, the querying of the complete dataset in a timely fashion is only possible on an optimized server with at least 192GB of RAM. It's clear that for larger pilots and commercial rollouts, more scalable databases are needed (e.g., commercial enterprise data management platforms or NoSQL solutions, e.g., mongoDB [21], Hadoop [22], Cassandra [23]), which is part of further research.

A dashboard tool was developed to monitor the incoming data and running experiments in real-time, and to inspect individual setups. This tool visualizes the captured data both per individual user and aggregated over groups of users and appliance types. It runs on top of a data warehouse, fed by aggregation scripts on the Data Server. As such it contains only the data that needs to be visualized, which improves the responsiveness. The data warehouse is updated by the aggregation scripts at the dominant frequency of the incoming data (every 15 minutes). As an example, Figure 6 shows the quarterly energy measurements for one household for one day. The tool can show similar graphs for the flexibility data generated by the smart appliances and smart meter measurements, including power and voltage.

\section{CONCLUSIONS}

In this paper we presented how we deployed the ICT infrastructure required for residential demand response with smart appliances in real life, focusing on the practical experiences during implementation and roll out.

The most critical technical issue we experienced, is the difficulty to realize a reliable in-house communication between gateway, measurement equipment and smart appliances. This problem needs to be addressed before a full scale roll-out of residential demand response becomes feasible. As a first step, two industrial partners of Linear already replaced their communication technology in their latest products to improve integration in existing houses. In a full scale roll-out adequate back-end processing power will be needed to process the huge 


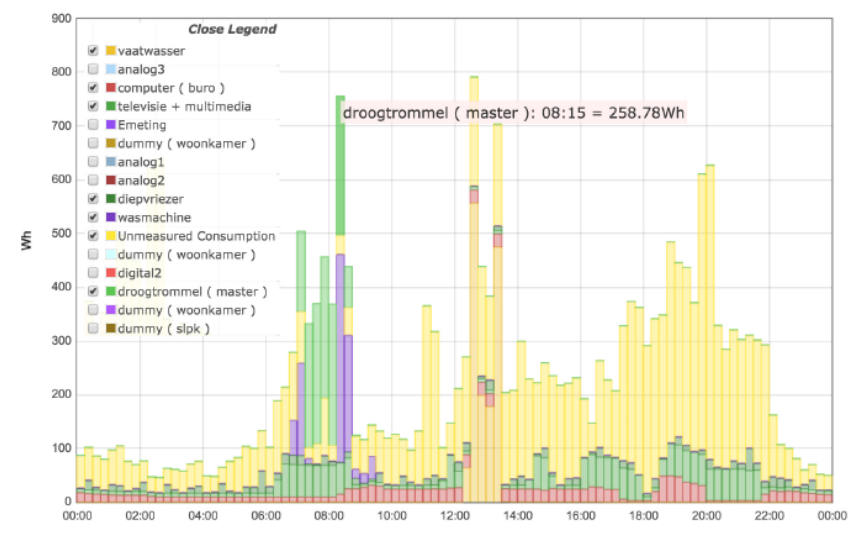

Fig. 6. Detailed consumption measurements for one household for one day.

amounts of data generated in real-time, and required for the optimal control of the smart appliances. Cloud services could be used for this purpose. Another important requirement for a full scale rollout, which was successfully addressed within Linear, is interoperability, both at the household level where a good device abstraction is needed and for the backend where multiple parallel providers with proprietary and differing control technology need to be supported. The concurrent support of balancing and congestion management business cases is still an open issue and needs further research.

At the end of 2014 all the collected data was processed and analyzed resulting in an assessment of the flexibility potential of residential consumption and the technical and economical viability of the researched business cases. All results were published in a booklet [24]. A first important conclusion is that flexibility offered by DHW buffers and especially by whitegoods is asymmetric: at any moment the power consumption can be increased much more than decreased. So e.g. for the wind balancing case we got good results when there was a surplus of wind energy compared to what was predicted, but realizing a negative power difference was much more difficult. Furthermore we noticed that the flexibility potential per household of a DHW buffer is 10 times bigger than the potential of the whitegoods.

The control of the flexible loads based on the dynamic tariffs resulted in a significant shift of consumption towards the cheap periods (afternoon and night). However, this flexible share remained small compared to the non-flexible load. Likewise, for the technical business cases, the locally available flex was often too small compared to the total local consumption in a neighbourhood to have a significant impact on the line voltages and transformer load.

Finally we noticed that users that did not have smart appliances and thus were asked to shift their consumption manually based on the (complex) day ahead dynamic tariffs showed a large response fatigue. On the other hand, people with smart appliances that simply had to provide flexibility by indicating a deadline on their devices when configuring them and that were rewarded using a capacity fee, showed no response fatigue. A large amount of these people indicated that they developed new routines during the pilot period, e.g., filling the dishwasher after dinner and providing flexibility until the next morning.

\section{ACKNOWLEDGMENT}

This work was supported by the Ministry of Science of Flanders via the project Linear organized by the Institute for Science and Technology (IWT).

\section{REFERENCES}

[1] E. Peeters, C. Develder, J. Das, J. Driesen, and R. Belmans, "Linear: towards a breakthrough of smart grids in flanders," in Proc. 2nd Int. Conf. Innovation for Sustainable Production (i-SUP 2010), vol. 3, Bruges, Belgium, 18-21 Apr. 2010, pp. 3-6.

[2] R. Belhomme, R. Cerero, G. Valtorta, and P. Eyrolles, "The address project: Developing active demand in smart power systems integrating renewables," in Power and Energy Society General Meeting, 2011 IEEE, July 2011, pp. 1-8.

[3] A. Faruqui and S. Sergici, "Household response to dynamic pricing of electricity: a survey of 15 experiments," Journal of Regulatory Economics, vol. 38, no. 2, pp. 193-225, 2010.

[4] K. Herter, Y. Okunevo, and V. Wood, "Smud's residential summer solutions, ibc," Herter Energy Solutions, Tech. Rep., January 2014.

[5] R. Kamphuis, B. Roossien, M. Eijgelaar, H. de Heer, J. van de Velde, and A. van den Noort, "Real-time trade dispatch of a commercial VPP with residential customers in the powermatching city smartgrid living lab," in Electricity Distribution (CIRED 2013), 22nd International Conference and Exhibition on, June 2013, pp. 1-4.

[6] S. Lannez, G. Foggia, M. Muscholl, J. Passelergue, C. Lebosse, and K. Mercier, "Nice grid: Smart grid pilot demonstrating innovative distribution network operation," in IEEE PowerTech (POWERTECH) Grenoble, June 2013, pp. 1-5.

[7] H. Saele and O. Grande, "Demand response from household customers: Experiences from a pilot study in norway," Smart Grid, IEEE Transactions on, vol. 2, no. 1, pp. 102-109, March 2011.

[8] C. Woo, R. Li, A. Shiu, and I. Horowitz, "Residential winter kwh responsiveness under optional time-varying pricing in British Columbia," Applied Energy, vol. 108, no. C, pp. 288-297, 2013.

[9] "Toyota city low-carbon society verification project," http://jscp.nepc.or.jp/en/toyota.

[10] "Ecogrid EU: From design to implementation," http://ecogridbornholm.dk/wp-content/uploads/2013/10/EcoGridReport-Octobre-2013.pdf, October 2013.

[11] "Your energy moment project (jouw energie moment)," http://jouwenergiemoment.nl/.

[12] “Greenlys project," http://www.greenlys.fr/.

[13] M. Strobbe, K. Vanthournout, T. Verschueren, W. Cardinaels, and C. Develder, "Large-scale residential demand response ict architecture," in Proc. of the 5th IEEE PES Innovative Smart Grid Technologies (ISGT) European Conference, October 2014.

[14] B. Dupont, J. Tant, and R. Belmans, "Automated residential demand response based on dynamic pricing," in Innovative Smart Grid Technologies (ISGT Europe), 2012 3rd IEEE PES International Conference and Exhibition on, October 2012, pp. 1-7.

[15] KNX Association, "KNX," http://www.knx.org.

[16] Netbeheer $\quad$ Nederland, "DSMR
http://www.netbeheernederland.nl/themas/hotspot/hotspotdocumenten/?dossierid $=11010056$.

[17] “MySQL Replication," http://dev.mysql.com/doc/refman/5.6/en/replication.html.

[18] V. T. Baron Schwartz, Peter Zaitsev, Ed., High Performance MySQL, 3rd Edition. O'Reilly Media, 2012.

[19] "Zabbix - The Enterprise Monitoring Solution for Everyone," http://www.zabbix.com/.

[20] R. Olups, Ed., Zabbix 1.8 Network Monitoring. Packt Publishing, 2010.

[21] “mongoDB," http://www.mongodb.org/.

[22] “Apache Hadoop," http://hadoop.apache.org/.

[23] “Apache Cassandra," http://cassandra.apache.org/.

[24] Linear: Demand Response for Families. Linear consortium, 2014. 